\title{
Tratamiento laparoscópico de un quiste de colédoco gigante en un paciente pediátrico
}

\author{
Carlos García-Hernández ${ }^{1,2 *}$, Lourdes Carvajal-Figueroa ${ }^{1,2}$, Christian Archivaldo-García ${ }^{1,2}$ y \\ Ariadna A. Alvelais-Arzamendi1,2 \\ ${ }^{1}$ Departamento de Cirugía Pediátrica, Hospital Infantil Privado; ${ }^{2}$ División de Estudios de Posgrado, Facultad de Medicina, Universidad Nacional \\ Autónoma de México. Ciudad de México, México
}

\begin{abstract}
Resumen
Introducción: Se define como quiste de colédoco gigante aquel con un diámetro $\geq 10 \mathrm{~cm}$. A pesar de que el abordaje laparoscópico ha sido contraindicado, se presenta el caso de un adolescente con un quiste de colédoco gigante resuelto por laparoscopía. Caso clínico: Paciente de sexo masculino de 14 años con un quiste de colédoco gigante tratado con anastomosis hepático-duodenal laparoscópica. Conclusiones: El tamaño promedio de los quistes de colédoco tratados por laparoscopía es de $40 \mathrm{~mm}$. No se recomienda la resección de quistes gigantes por mínima invasión debido a adherencias y restricción del campo visual. En este caso se realizó un tratamiento laparoscópico de manera exitosa.
\end{abstract}

Palabras clave: Quiste de colédoco gigante. Reconstrucción bilioentérica. Laparoscopía. Anastomosis hepático-duodenal. Pediatría.

\section{Laparoscopic treatment of a giant choledochal cyst in a pediatric patient}

\section{Abstract}

Background: The giant choledochal cyst has a diameter $\geq 10 \mathrm{~cm}$. Although laparoscopy has been contraindicated, we present the case of a teenager with a giant choledochal cyst resolved by laparoscopy. Case report: A 14-year-old male patient with a giant choledochal cyst treated with hepatic-duodenum laparoscopic anastomosis. Conclusions: The average size of bile duct cysts treated by laparoscopy is $40 \mathrm{~mm}$. Giant cysts should not be resected through minimal invasion due to adhesions and a restricted visual field. We report a case of a giant cyst successfully treated by laparoscopy.

Keywords: Giant choledochal cyst. Biliary reconstruction. Laparoscopy. Hepatic-duodenum anastomosis. Pediatrics.

\section{Introducción}

El quiste de colédoco es una rara afección benigna de la vía biliar ${ }^{1,2}$. Debido a la posibilidad de infectarse o malignizarse, el principio del tratamiento es la resección del colédoco con reconstrucción biliodigestiva ${ }^{1}$.
Aunque se desconoce su etiología, una de las teorías sobre su formación más ampliamente aceptadas es la anomalía en la unión pancreatobiliar que permite el reflujo de enzimas en el árbol biliar y la obstrucción del conducto biliar común ${ }^{3-5}$.
Correspondencia:

*Carlos García Hernández

E-mail: carloscirped@ hotmail.com
Fecha de recepción: 8-06-2020

Fecha de aceptación: 24-07-2020

DOI: 10.24875/BMHIM.20000149
Disponible en internet: 02-06-2021 Bol Med Hosp Infant Mex. 2021;78(4):350-355

www.bmhim.com 1665-1146/@ 2020 Hospital Infantil de México Federico Gómez. Publicado por Permanyer. Este es un artículo open access bajo la licencia CC BY-NC-ND (http://creativecommons.org/licenses/by-nc-nd/4.0/). 
El tratamiento puede llevarse a cabo mediante cirugía laparoscópica, como publicaron por primera ocasión Farello et al. ${ }^{6}$, quienes operaron a una niña de 6 años con buen resultado. Actualmente existen numerosos trabajos que reportan la posibilidad de llevar a cabo este procedimiento mediante mínima invasión ${ }^{7-9}$. Sin embargo, existen pocas publicaciones que discutan la importancia del tamaño del quiste para decidir el tipo de abordaje quirúrgico ${ }^{3}$. Solo algunos reportes de tratamientos por mínima invasión mencionan el tamaño de los quistes, que en promedio es $<5 \mathrm{~cm}^{10,11}$. No obstante, el tamaño del quiste no debe ser impedimento para realizar un abordaje laparoscópico ${ }^{12}$.

Un quiste de colédoco se considera como gigante ${ }^{3}$ si tiene un diámetro $\geq 10 \mathrm{~cm}$. Los quistes gigantes son diferentes de los de menor tamaño no solo en sus dimensiones, sino porque presentan otras características que dificultan su tratamiento; entre ellas, destaca el haber cursado con un mayor número de cuadros de colangitis aguda, por lo que presentan más adherencias que, a su vez, condiciona un mayor sangrado durante la resección del quiste. A causa del gran tamaño del quiste, la restricción del campo visual también dificulta el tratamiento por mínima invasión ${ }^{3}$.

Por todo lo anterior, el abordaje laparoscópico no se ha recomendado para los pacientes con quistes de colédoco gigantes $\geq 10 \mathrm{~cm}$. Incluso, en algunos reportes se menciona que el tamaño de la cavidad dificulta la disección y el tratamiento laparoscópico ${ }^{13,14}$. Asimismo, algunas publicaciones mencionan que se requiere la conversión a cirugía abierta para los quistes gigantes ${ }^{15}$.

Los reportes de tratamiento laparoscópico de quistes de colédoco gigantes son escasos. Sin embargo, llama la atención la publicación de Diao et al. ${ }^{16}$, que demuestra la posibilidad de realizar este tratamiento en pacientes pediátricos mediante cirugía laparoscópica de una sola incisión ${ }^{16}$.

El objetivo del presente trabajo fue reportar el caso de un paciente pediátrico con un quiste de colédoco gigante resuelto de manera exitosa por cirugía de mínima invasión.

\section{Caso clínico}

Se describe el caso de un paciente de sexo masculino de 14 años sin antecedentes de importancia. Inició su padecimiento actual 2 meses antes de su ingreso, con ictericia acompañada de coluria y acolia de 5 días de duración que remitieron sin tratamiento. Posteriormente presentó múltiples episodios de dolor abdominal de tipo cólico, que cedieron con la administración de analgésicos.

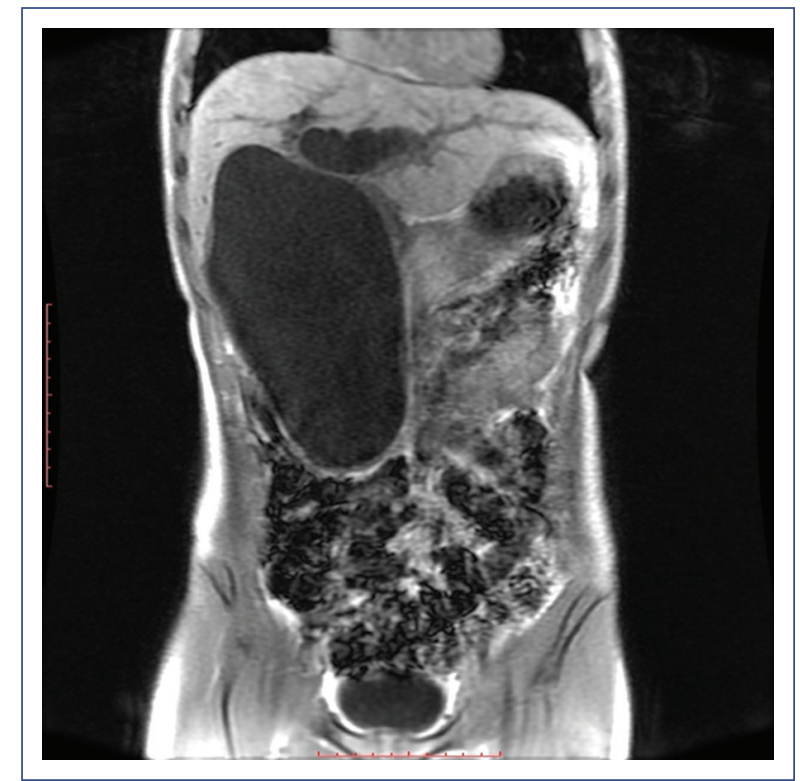

Figura 1. Resonancia magnética ponderada en T2. Se observa una imagen hipointensa con morfología fusiforme y comportamiento de contenido líquido en relación con un quiste de colédoco.

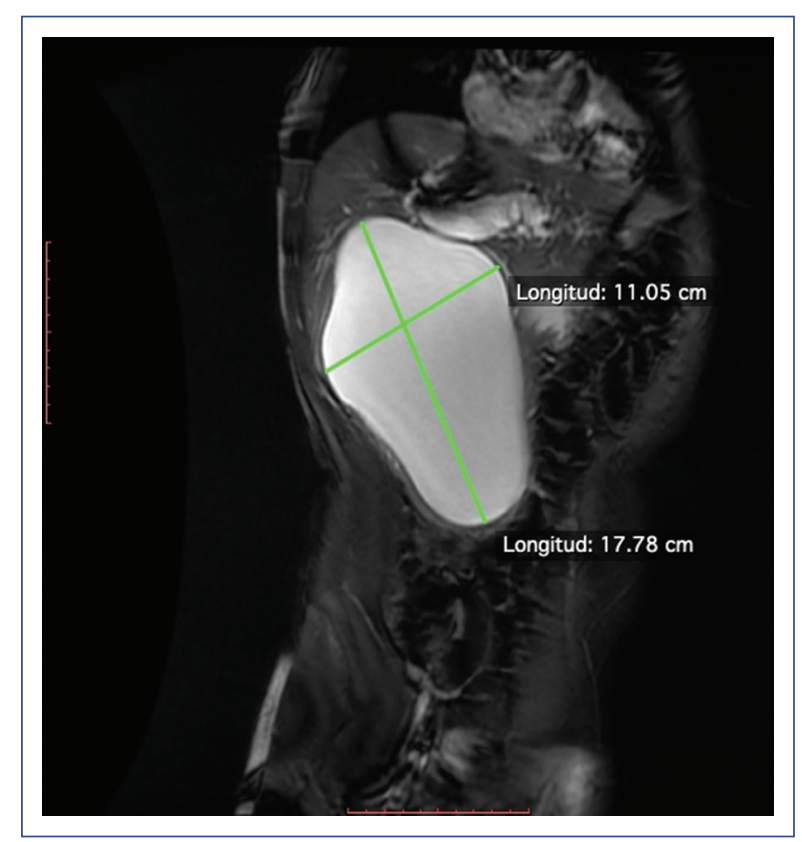

Figura 2. Corte coronal en secuencia T2. Se observa una imagen hiperintensa de morfología fusiforme, ubicada en el espacio de Morrison, que condiciona desplazamiento lateral de la cabeza del páncreas y se acompaña de dilatación de la vía biliar intrahepática.

Quince días antes de su ingreso presentó distención abdominal acompañada de un nuevo episodio de ictericia. En la exploración física se observó tinte ictérico 


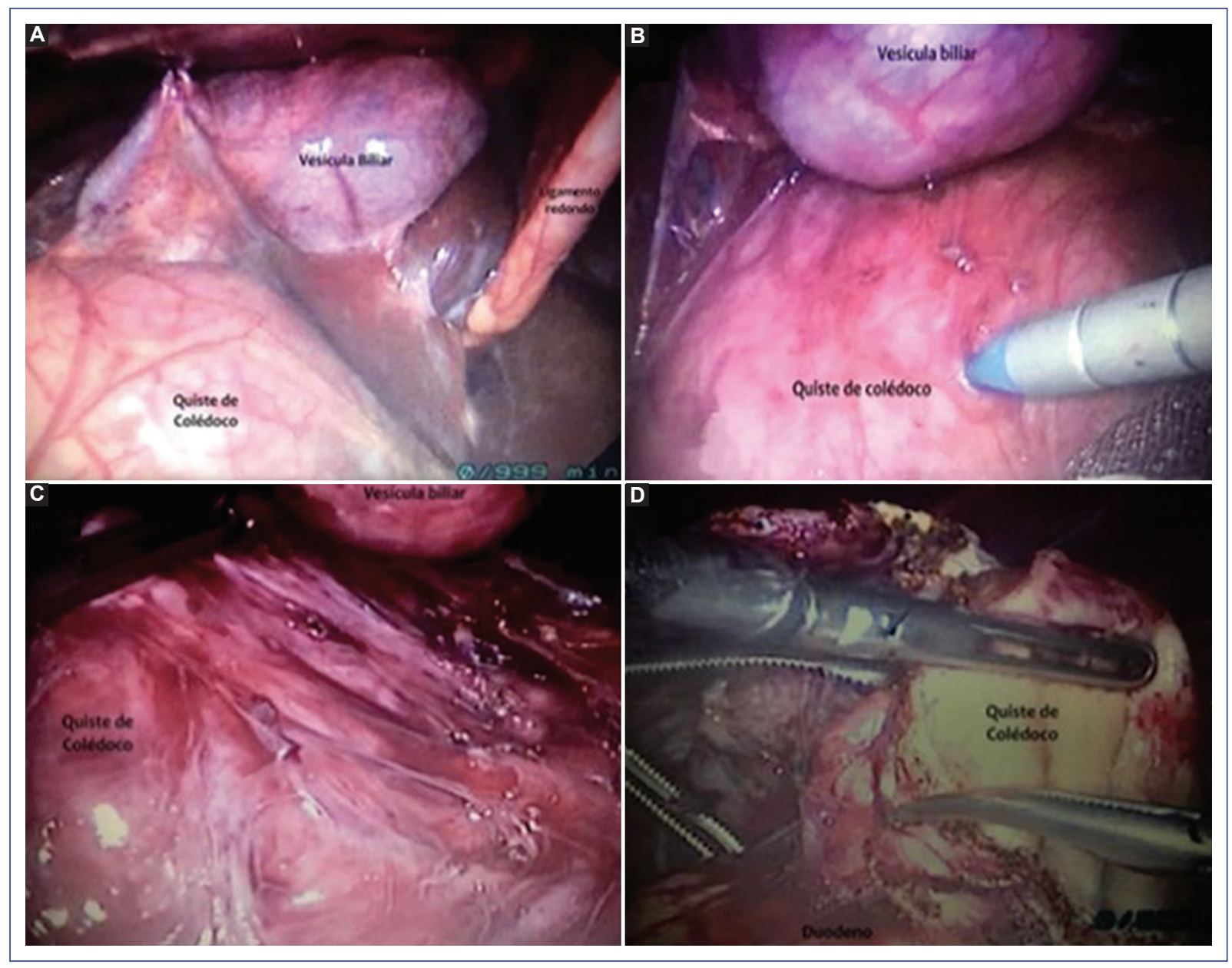

Figura 3. A: se aprecian el colédoco dilatado y la vesícula biliar distendida y tensa, que se fija a la pared para favorecer la exposición del quiste. B: punción del quiste para facilitar su disección. C: después de la punción se facilita la disección de la vena porta hasta separarla del quiste. D: sección del quiste del lado duodenal, donde se aprecia el gran diámetro del colédoco.

en las conjuntivas y a la palpación abdominal se encontró una tumoración móvil, dura, de bordes regulares, desde el reborde costal derecho hasta la fosa ilíaca homolateral, no dolorosa, que rebasaba la línea media. Los estudios de laboratorio mostraron elevación de la bilirrubina total $(4.5 \mathrm{mg} / \mathrm{dl})$, predominio de la bilirrubina directa $(3.7 \mathrm{mg} / \mathrm{dl})$ y fosfatasa alcalina de $750 \mathrm{U} / \mathrm{l}$. El resto de los resultados se encontraban dentro de los límites normales (proteínas totales $7.2 \mathrm{~g} / \mathrm{dl}$, transaminasa glutámica oxalacética $24 \mathrm{U} / \mathrm{l}$, transaminasa pirúvica $26 \mathrm{U} / \mathrm{l}$, gamma glutamil transpeptidasa $52 \mathrm{U} / \mathrm{l}$, amilasa sérica $63 \mathrm{U} / \mathrm{ly}$ lipasa sérica $143 \mathrm{U} / \mathrm{l})$. El ultrasonido mostró una gran masa quística localizada en el hipocondrio derecho; en la topografía del colédoco se encontró dilatación de la vía biliar intrahepática. Se realizó una colangiorresonancia que mostró dilatación de la vía biliar intrahepática y extrahepática
(Fig. 1), dilatación fusiforme del colédoco con dimensiones de $11.05 \times 17.78 \mathrm{~cm}$ y pared de $2.4 \mathrm{~mm}$, dilatación hasta el tercio distal de los conductos hepáticos derecho e izquierdo, sin compromiso de la vena porta ni de la cava (Fig. 2). Se consideró que se trataba de un quiste de colédoco gigante tipo Ic de la clasificación de Alonso-Lej modificada por Todani et al. ${ }^{17}$ y se planeó su tratamiento quirúrgico.

Se procedió a realizar anastomosis hepático-duodenal con resección del colédoco mediante abordaje laparoscópico, con presión de $10 \mathrm{mmHg}$ y óptica de 30 grados. Se colocó un trocar de $10 \mathrm{~mm}$ con técnica abierta en la cicatriz umbilical para explorar la cavidad abdominal. Se identificó una dilatación quística gigante del colédoco que ocupaba gran parte de la cavidad abdominal, además de la vesícula biliar tensa. Se colocaron otro trocar de $10 \mathrm{~mm}$ y dos más 


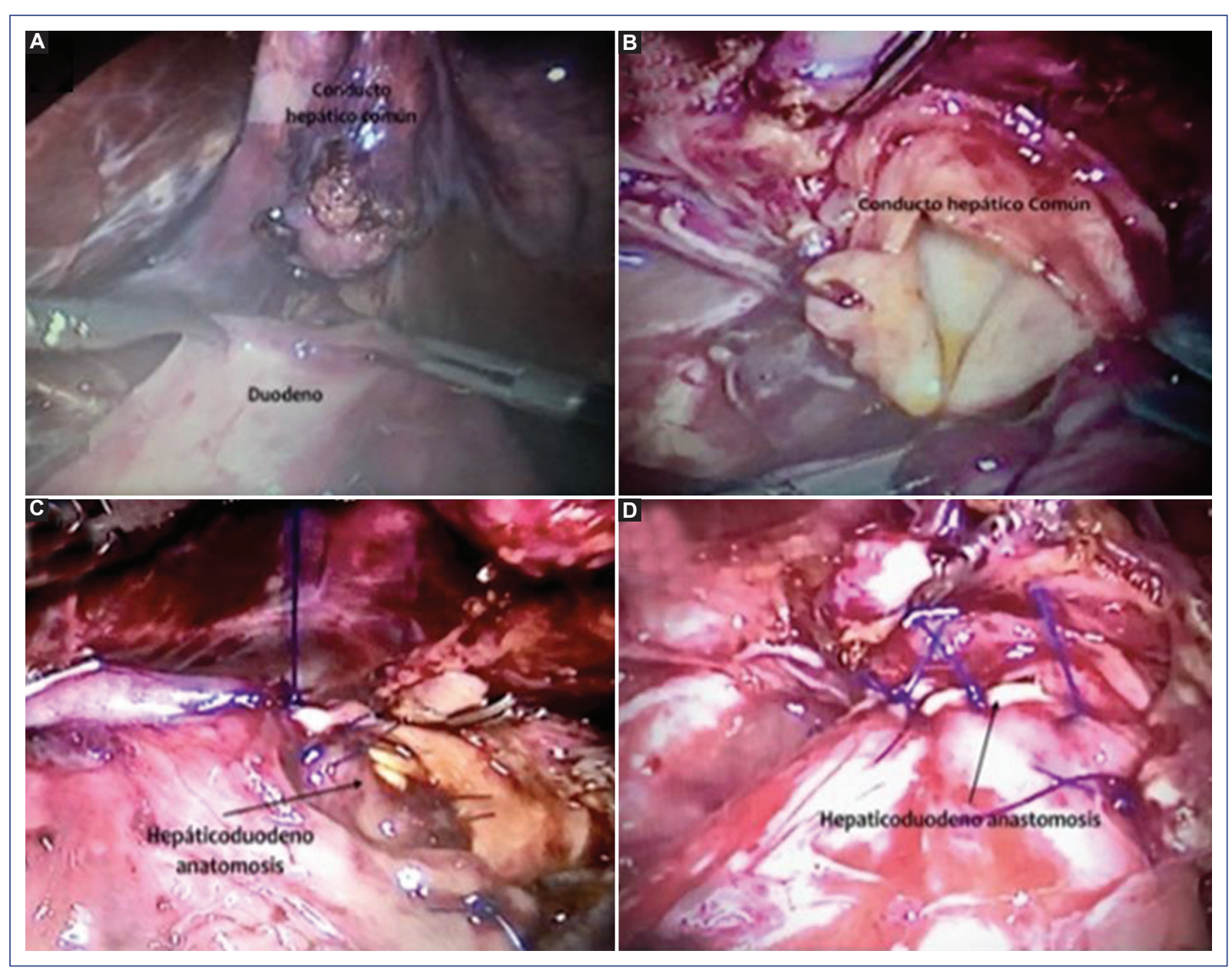

Figura 4. A: movilización del duodeno hacia el hilio hepático. B: exposición del conducto hepático común para preparar la anastomosis. C: anastomosis de la cara posterior del conducto hepático común con el duodeno. D: aspecto final de la anastomosis hepático-duodenal.

de $5 \mathrm{~mm}$ bajo visión directa. Se practicó una colangiografía de manera percutánea que demostróla desembocaduraal duodeno. Después se colocó un punto de tracción de la vesícula biliar a la pared abdominal, se puncionó de inmediato el quiste de colédoco y se drenaron $600 \mathrm{ml}$ de contenido biliar, lo que favoreció la disección del quiste. Posteriormente, se identificaron y separaron la vena porta y la arteria hepática del quiste. Se identificó y seccionó la unión del colédoco dilatado con el duodeno y se reparó el sitio de inserción duodenal con un surgete continuo de sutura de polidaxona 4-0. Se continuó la disección de manera ascendente hasta llegar al conducto hepático común, donde se realizó un corte transversal. Se ligaron el conducto y la arteria císticos (Fig. 3), y se resecó y extrajo el quiste. A continuación, se realizó kocherización del duodeno y una incisión a $3 \mathrm{~cm}$ del píloro para una anastomosis al conducto biliar común con puntos separados. Después se disecó y extrajo la vesícula biliar (Fig. 4), y se colocó un drenaje tipo Penrose por contrabertura. El aspecto macroscópico del hígado era normal, por lo que no se realizó biopsia hepática.

El sangrado fue de $200 \mathrm{ml}$ y el tiempo quirúrgico de 170 minutos, sincomplicaciones transoperatorias ni posoperatorias. Se inició la vía oral al cuarto día del posoperatorio y el paciente egresó en el sexto día posterior a la operación. Las cifras elevadas de bilirrubinas y fosfatasa alcalina se normalizarona los 15 días del posoperatorio. El resto de los parámetros de laboratorio se mantuvieron dentro de los límites normales. Alos 2 meses se realizó una panendoscopia de control, en la que se observó ausencia de lago biliar en el estómago y anastomosis funcional con salida de bilis en el duodeno (Fig. 5).

El paciente se encontró asintomático y con una buena evolución en el seguimiento clínico y con 


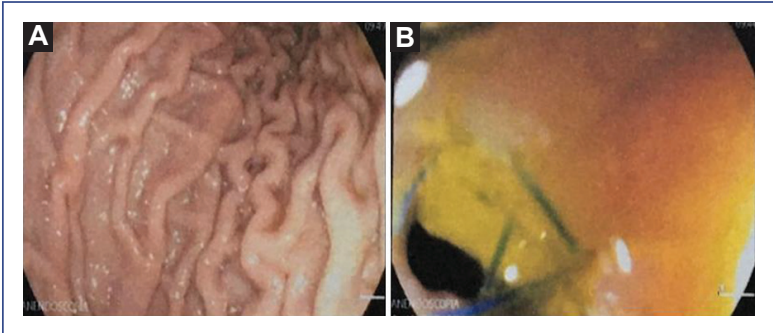

Figura 5. Imágenes endoscópicas al segundo mes del posoperatorio. A: se aprecian pliegues gástricos engrosados con ausencia de material biliar. B: salida de bilis por el sitio de la anastomosis a nivel duodenal. Se puede apreciar el material de sutura.

pruebas de función hepática normales 12 meses después la operación.

\section{Discusión}

El quiste de colédoco es una patología poco frecuente. Debido a la posibilidad de malignización o de infección, se debe resecar y llevar a cabo una reconstrucción bilioentérica ${ }^{2}$. Numerosos reportes han demostrado las bondades de la cirugía de mínima invasión en esta situación ${ }^{1,7-10}$. Sin embargo, el tamaño promedio del quiste ${ }^{10}$ que se ha mencionado es de $40 \mathrm{~mm}$. Se consideran quistes gigantes aquellos $\geq 10 \mathrm{~cm}$ y se ha sugerido que no deben resecarse por mínima invasión, ya que presentan una mayor cantidad de adherencias y el campo visual está restringido por su tamaño $0^{3,13}$.

En el presente caso se abordó porlaparoscopía un quiste de $11 \mathrm{~cm}$ de diámetro. Se considera que esto fue posible debido a los siguientes factores:

- Por la colocación de los trocares de acuerdo con la topografía del quiste, determinada en los hallazgos transoperatorios.

- Por el drenaje inmediato del quiste, lo que facilitó la disección de la vena porta y de la arteria hepática, además de un mayor espacio de trabajo.

- Por el cierre de la desembocadura del quiste al duodeno con un surgete continuo debido a su gran tamaño.

La reconstrucción de la vía biliar mediante anastomosis hepático-yeyunal en $Y$ de Roux 0 anastomosis hepático-duodenal (como en este paciente) ha demostrado los mismos resultados que la anastomosis al yeyuno en términos de control de la enfermedad. Sin embargo, es más fácil de realizar, ya que solo requiere una anastomosis, aunque la gran dilatación del conducto hepático común facilitó esta reconstrucción.
El diagnóstico de un quiste gigante de colédoco no debe considerarse una contraindicación para el tratamiento laparoscópico, ya que, con las recomendaciones mencionadas, este procedimiento puede llevarse a cabo de manera segura y ofrecer al paciente las ventajas de la mínima invasión.

\section{Responsabilidades éticas}

Protección de personas y animales. Los autores declaran que para esta investigación no se han realizado experimentos en seres humanos ni en animales.

Confidencialidad de los datos. Los autores declaran que han seguido los protocolos de su centro de trabajo sobre la publicación de datos de pacientes.

Derecho a la privacidad y consentimiento informado. Los autores han obtenido el consentimiento informado de los pacientes o sujetos referidos en el artículo. Este documento obra en poder del autor de correspondencia.

\section{Conflicto de intereses}

Los autores declaran no tener conflictos de intereses.

\section{Financiamiento}

Ninguno.

\section{Bibliografía}

1. Chokshi NK, Guner YS, Aranda A, Shin CE, Ford HR, Nguyen NX. Laparoscopic choledochal cyst excision: lessons learned in our experience. J Lap Adv Surg Tech. 2009;19:87-91.

2. de Vries JS, de Vries S, Aronson DC, Bosman DK, Rauws EAJ, Bosma A, et al. Choledochal cysts: age of presentation, symptoms, and late complications related to Todani's classification. J Pediatr Surg. 2002;37:1568-73.

3. Anand U, Priyadarshi RN, Kumar B, Khandelwal C. Diagnosis and management of giant choledochal cysts: complexities compared to smaller cysts. Indian J Gastroenterol. 2013;32:262-7.

4. Cha SW, Park MS, Kim KW, Byun JH, Yu JS, Kim MJ, et al. Choledochal cysts and anomalous pancreaticobiliary ductal union in adults: radiological spectrum and complications. J Comput Assist Tomogr. 2008;32:17-22.

5. Gezer HÖ. Pediatric choledochal cysts: unknowns are decreasing. En: Sameh S, editor. Pediatric surgery, flowcharts and clinical algorithms. Londres: IntechOpen; 2019. p. 1-27.

6. Farello GA, Cerofolini A, Rebonato M, Bergamaschi G, Ferrari C, Chiappetta A. Congenital choledochal cyst: video-guided laparoscopic treatment. Surg Laparosc Endosc. 1995:5:354-8.

7. Ure BM, Schier F, Schmidth AL, Nustede R, Petersen C, Jesch NK. Laparoscopic resection of congenital choledochal cyst choledochojejunostomy, and extra abdominal Roux-en-Y anastomosis. Surg Endosc. 2005;19:1055-7.

8. Lee H, Hirose S, Bratton B, Farmer D. Initial experience with complex laparoscopic biliary surgery in children: biliary atresia and choledochal cyst. J Pediatr Surg. 2004;39:804-7.

9. Aspelund G, Ling SC, Ng V, Kim PA. A role for laparoscopic approach in the treatment of biliary atresia and choledochal cysts. J Pediatr Surg. 2007:42:869-72.

10. Liem NT, Pham HD, Dung LA, Son TR, Vu HM. Early and intermediate outcomes of laparoscopic surgery for choledochal cysts with 400 patients. J Laparoendosc Adv Surg Tech A. 2012;22:599-603. 
11. Narayanan SK, Chen Y, Narasimhan KL, Cohen CR. Hepaticoduodenostomy versus hepaticojejunostomy after resection of choledochal cyst: a systematic review and meta-analysis. J Pediatr Surg. 2013;48:2336-42.

12. Chan EKW, Lee KH, Wong VHY, Tsui BSY, Wong BS, Pang KK, et al. Laparoscopic management of choledochal cyst in infants and children: a review of current practice. Surg Pract. 2018;22:131-7.

13. Yeung F, Fung ACH, Chung PHY, Wong KKY. Short-term and long-term outcomes after Roux-en-Y hepatic jejunostomy versus hepaticoduodenostomy following laparoscopic excision of choledochal cyst in children. Surg Endosc. 2020;34:2172-7.
14. Koo EJ, Jung E, Choi SO. Laparoscopic choledochal cyst excision and hepaticojejunostomy: a case series. J Min Inv Surg. 2017;20:58-62.

15. Hong L, Wu HY, Yan Z, Xu M, Chu J, Chen QM. Laparoscopic surgery for choledochal cyst in children: a case review of 31 patients. Eur $\mathrm{J}$ Pediatr Surg. 2008;18:67-71.

16. Diao M, Li L, Li Q, Ye M, Cheng W. Challenges and strategies for single-incision laparoscopic Roux-en-Y hepaticojejunostomy in managing giant choledochal cysts. Int J Surg. 2014;12:412-7.

17. Todani $T$, Watanabe $Y$, Narusue M, Tabuchi K, Okajima K. Congenital bile duct cysts. Classification, operative procedures and review of thirty-seven cases including cancer arising from choledochal cyst. Am J Surg. 1977;134:263-9. 